\title{
The Application of Service-Oriented Information Sharing Program in Geographic Information System
}

\author{
Han Zhang ${ }^{1, a}$, Jianhong $\mathrm{Ma}^{1, \mathrm{~b}}$ \\ ${ }^{1}$ Software Technology School, ZhengZhou University, 450002, China \\ a email: tianyadehui@tom.com ${ }^{\text {b }}$ email:majianhong@zzu.edu.cn
}

Keywords: Distributed Geographic Information Sharing, Web Services, GML, XSLT

\begin{abstract}
Geospatial information with the feature of multi-source, multi-space-time, multisemantics, multi-scale and diversity of data formats, makes geospatial information sharing a complex issue, For this reason, to resolve spatial information sharing is the key to spatial information applications and services. This paper focuses on geospatial information sharing technology, presenting a service-oriented geographic information sharing framework, a better solution for distributed geospatial information sharing. The main word includes:Introducing the current development of Geospatial information technology and spatial information data storing, and deep exploring service-oriented concept of the geographical information sharing. And gives specific application in system
\end{abstract}

\section{Introduction}

The diversity of GIS and spatial information systems determine the characteristics of multisource geographic information, and more space, more than semantics, multi-scale and diversity of data formats, allowing the sharing of spatial information into a very complex issue. To this end, solve the problem of spatial information sharing is the key to spatial information applications and services. Distributed storage of geographic information can only retrival and process the information stored in local database, but can't make use of the data in other geographic information system nor organize and manage the geographic information efficiently.In order to achieve cross-platform data sharing and application development, It must be done a lot of different format conversion work.for different kinds of data sources. Because of these problems, geographic information format conversion and sharing of geographic information systems become a hot issue. Now we propose a new geographic information sharing program that is service-oriented geographic information sharing program.

\section{Service-oriented geographic information sharing program}

Service-oriented geographic information sharing basic idea is different GIS should follow Other service users or geographical information system by calling the Web service interface to access to geographical data which its services can provide ,between different Web services can also directly call the service interface to interact with each other

1) Geographic Information conversion based on GML

GML is the standard of spatial data exchange format. There are three basic XML Schema consists of it, which feature.xsd defines an abstract model of geographical features, geometry.xsd defines the specific geometric shape information, xlink.xsd defines various functions link. The three schema is equivalent to three base classes. Though inheritance and extensions of these three schema can define their own schema to encode geographic data in order to achieve the manner of XML and model geographic information in WebGIS.

2) Principle of data service layer

Different geographic information systems in accordance with international standards of organizations to develop their own Web services. Other geographic information system or clientside to access geographic data by calling Web service interface, between different Web services can call 
the service interface to interact with each other. The Web services shield differences in the underlying data structure of the geographic information system, but also enables users to access through the network to more types, wider range of geographic information.

As a data service layer, based on the above criteria, according to the need to implement Web Map Service, Web Feature Service and Web Coverage Service in one or all of these. As a distributed geographic information sharing, the focus of this thesis is the sharing of geographic data,rather than web services or web coverage. The paper focuses on web feature services, through the realization of web feature services provided to the outside world of geographic information in GML format for the target use of geographic information systems, in order to achieve distributed geographic information sharing

\section{Design of Distributed Geographic Information Sharing System}

GML package geographic data, is responsible for geographic data sharing and transfer graphics to explain to the client application. Customer responsible for the interpretation of the data formats, including SVG, VRML and X3D, etc. Geographic Information Services is responsible for transforming the data into the GML format data format for the target system. That is distributed heterogeneous GIS system to provide a unified service interface to the outside to provide geographic information services of GML format. Geographic Information Transformation Services, responsible for the data into GML format for the target system to support geographic data formats, the target system is responsible for parsing and display the geographic information.

Based on the above analysis, the paper presents the concept of service-oriented based on geographic information-sharing architecture: Each spatial information system provides a standard Web services of GML format to the outside. Then the Web applications according to different GIS clients transform the GML format data into the corresponding target data format for analysis and disply of the client, in order to achieve a conversion, you can integrate all formats of GIS data, and reduce the frequency conversion data distortion.

As shown below, is the overall system architecture diagram:

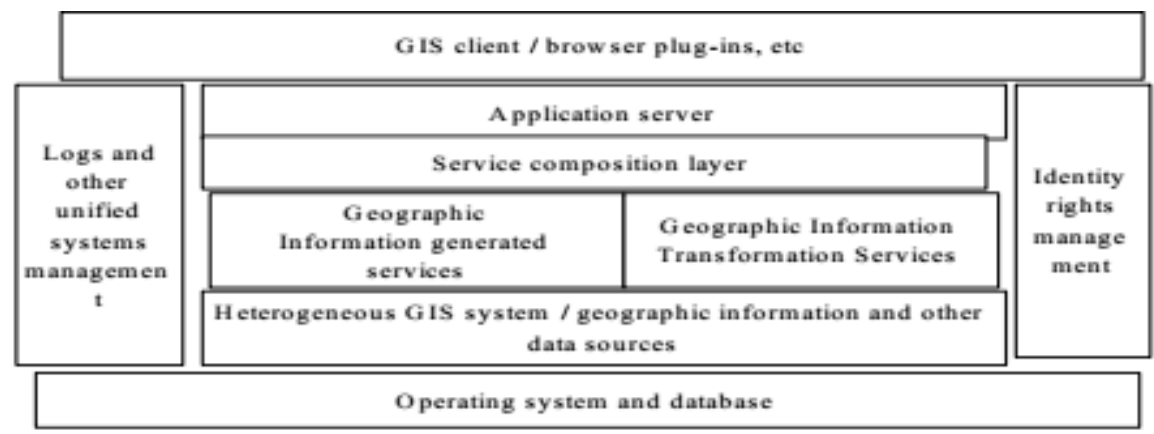

Fig. 1: Service-Oriented Geographic Information Sharing Architecture chart

The figure shows the author's distributed geographic information sharing architecture. The share structure of both unity and diversity of the underlying GIS data, but also provide services for a variety of GIS. Allows each module to achieve loosely coupled, shielding the underlying implementation, hiding the underlying complexity, Upper transparent geographical information users can easily access data sources across complex geographical data distributed to achieve a good geographic information sharing.

A number of distributed GIS to the outside world to provide a unified Web service interface, the Web service, responsible for customer requests, dynamically generate and package for GML file or string of geographic information package. Geographic information Conversion Service, is responsible for responding to the top customer requests, calling the bottom of a Web service, will it generate the GML into the module into the target through the GIS data formats supported. In fact, the module can also be divided into two parts: one part is GML transforms into a geographic 
information data based on XML, another is GML into data of non-XML format. Specific calls which module, depending on the target GIS.

\section{Data services layer design}

The XSLT specification defines an XML-based language used to express a kind of XML document into another type. The traditional XSTL is used to parse GML document into a DOM tree and then matches the style sheet file. But this growing practice in the case of GML data, the processor will be out of memory error.

By comparing the XMl parsing technology, DOM parsing requires a lot of memory, does not apply to a large amount of GML documents parsing. SAX parsing events by controlling access to XML documents, the order of events to promote the resolution in accordance with the document, there is the disadvantage of DOM parsing, SAX parsing controls accessing by events of XML documents,promote to parse orderly, this can be avoid disadvantage of DOM; STAX resolved by the application to control, selectively pull out the required contents, the document more user-friendly for special needs

Therefore, this system combines the advantages of different XML parsing technology, the source GML document using SAX parsing, to ensure the conversion of the large mount of document requests while parsing XSL stylesheet with stax, to ensure efficiency. The specific design shown in Figure 2

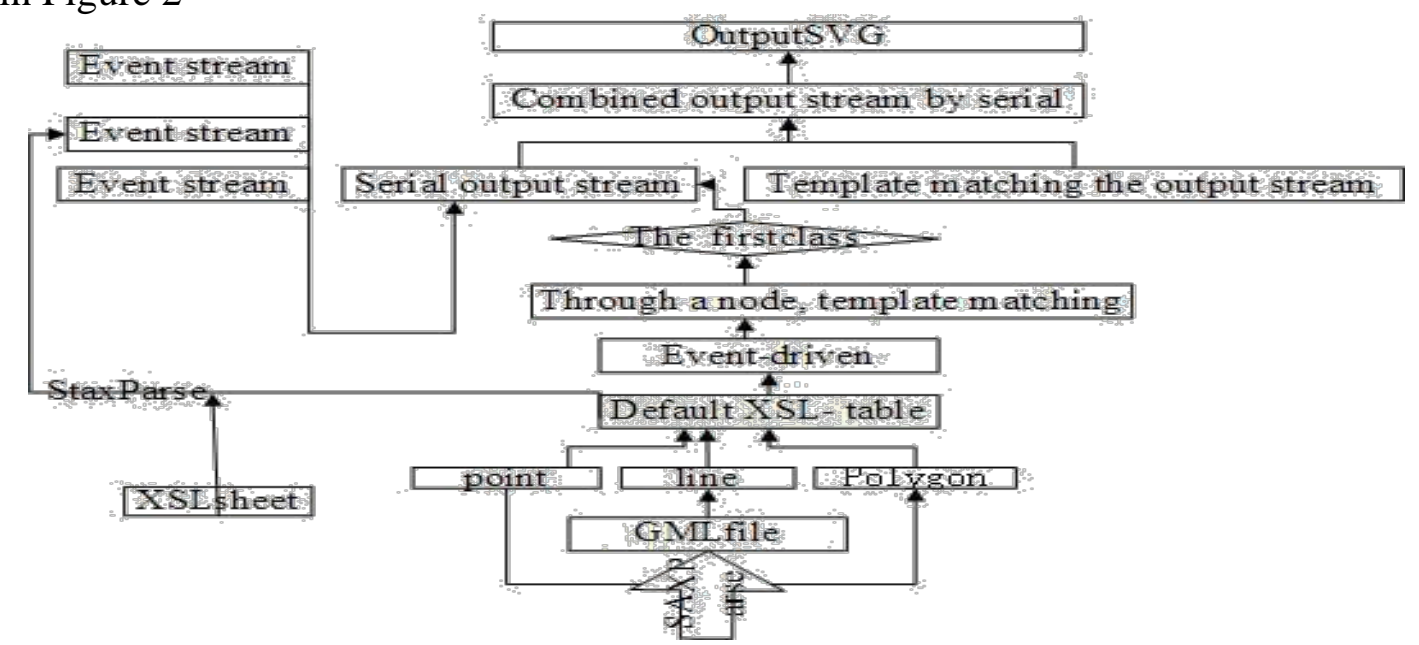

Fig.2 data layer Architecture chart

\section{Web services in the specific application in the system}

Web services layer consists of two parts: one is the generation of geographic information services, another is a conversion service. They are responsible for the GIS system will be heterogeneous in different formats of GIS data in GML format to return the caller to the service

1) Geographic information services generated

The Web service is responsible to respond to system calls, to return relevant geographic information by user searching and generate underlying GML file,which use for upper format conversion module. Provides the following interfaces: 


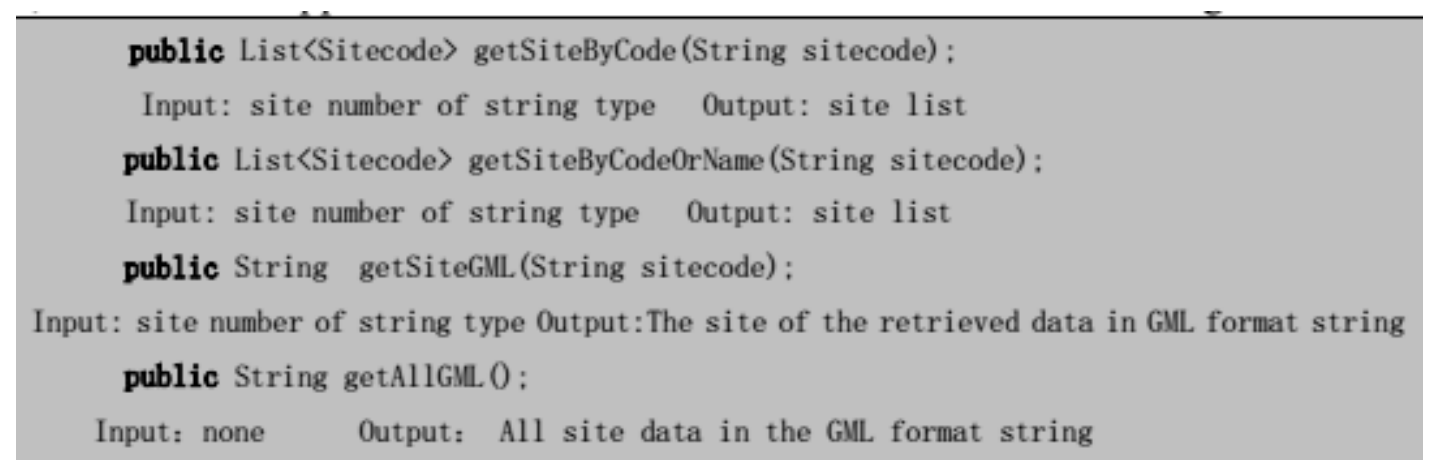

Fig.3 Interface code chart

2) Geographic Information Transformation Services

The Web service is responsible for the GML format file into the target system's data format. Conversion process has two pathways: GML to other types of XML format, GML format to convert non-XML type.specific to our system to achieve Just realize GML to XML type (specifically, the type of KML) format conversion pathways can be. With Process class in org.apache.xalan.xslt package,we can transform data of GML format to data of KML format by XSLT template. The following code shows the XML data format conversion sub-class service interface.

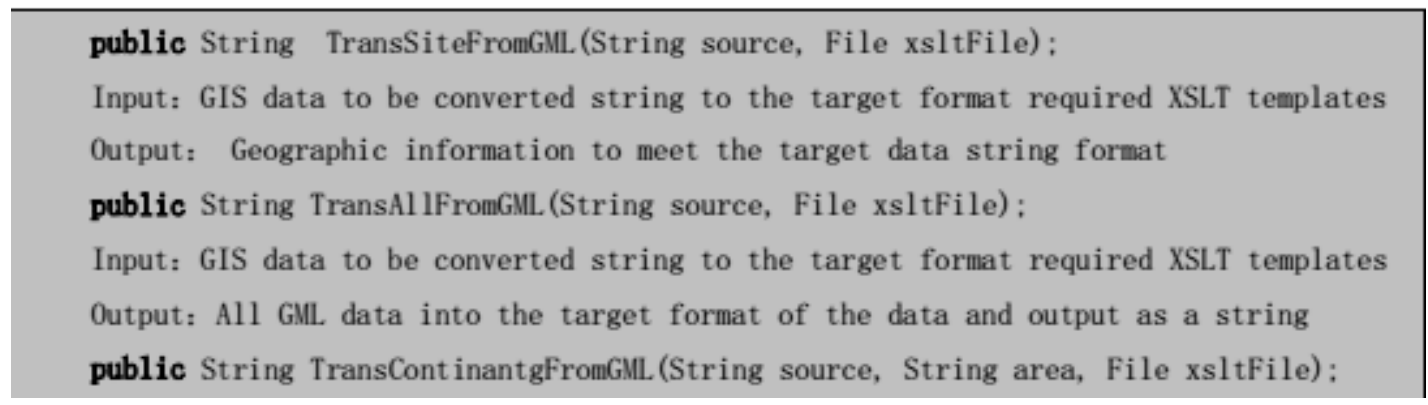

Fig.4 Interface code chart

\section{Conclusion}

This paper proposed a service-oriented geographic information sharing concept and from this concept we design a set of scientific and efficient framework for distributed geographic information sharing. Because of limited space, this article is only designed a conversion from GML to KML template, then we can write a set of template which GML to other XML-based format conversion, and further to perfect, more to reduce the loss caused by format conversion .

\section{References}

[1] ESRI,ESRI’s Approach to Interoperability,White Paper Series,1998

[2] Open GIS Consortium Inc, An Open GIS Consortium White Paper,2003

[3] Arliss Whiteside, OpenGIS Web Services Architecture Description,2005 [4]HuangHao Serviceoriented geographic information sharing to achieve on Google Earth 2007 